\title{
TEORÍA DE SITUACIÓN ESPECÍFICA DE FACTORES PROTECTORES SOCIO ECOLÓGICOS DEL CONSUMO DE ALCOHOL EN JÓVENES UNIVERSITARIOS
} SITUATION-SPECIFIC THEORY OF SOCIO-ECOLOGICAL PROTECTIVE FACTORS OF ALCOHOL CONSUMPTION IN YOUNG UNIVERSITY STUDENTS

\begin{abstract}
Pedro González-Angulo Universidad Juárez Autónoma de Tabasco, División Académica Multidisciplinaria de Jalpa de Méndez, Tabasco, México, estudiante de doctorado en ciencias de enfermería de la Universidad Autónoma de Nuevo León, Facultad de Enfermería, Monterrey, Nuevo León, México

María Magdalena Alonso-Castillo Universidad Autónoma de Nuevo León, Facultad de Enfermería, Monterrey, Nuevo León, México Nora Angélica Armendáriz-García Universidad Autónoma de Nuevo León, Facultad de Enfermería, Monterrey, Nuevo León, México Carla Aparecida Arena Ventura Universidade de São Paulo, Escola de Enfermagem de Ribeirão Preto. Ribeirão Preto, São Paulo, Brasil
\end{abstract}

Correspondencia:

María Magdalena Alonso Castillo (magdalena_alonso@hotmail.com)

Cómo citar este texto:

González-Angulo, P., Alonso-Castillo, M.M., Armendáriz-García, N.A. y Ventura, C.A.A. (2022). Teoría de Situación Específica de Factores Protectores Socio Ecológicos del Consumo de Alcohol en Jóvenes Universitarios. Health and Addictions / Salud y Drogas, 22(1), 167-186. doi: 10.21134/haaj. v22i1.646 


\title{
Resumen
}

Introducción: las Teorías de Situación Específica (TSE) abordan un fenómeno relativamente concreto y específico que permite describir, explicar y predecir como ocurre. Su principal función es proporcionar una estructura y una guía para la interpretación de comportamientos, procesos, respuestas humanas y eventos inicialmente desconcertantes. Objetivo: generar una TSE que explique los factores protectores socio ecológicos del consumo de alcohol en jóvenes universitarios, producto del análisis del Modelo Socio Ecológico (MSE) de McLeroy. Metodología: se utilizó la estructura Conceptual-Teórico-Empírico (C-T-E) propuesto por Fawcett, el cual consiste en cinco pasos 1) identificación de conceptos, 2) clasificación de los conceptos, 3) identificación y clasificación de las proposiciones, 4) orden jerárquico de proposiciones y 5) construcción del diagrama. Resultados: la TSE es el resultado de la estructura C-T-E, contribuye a la identificación de un fenómeno específico de la disciplina, determina conceptos, relaciones y posibles efectos de los factores protectores sobre la conducta del consumo de alcohol. Conclusión: el método fue apropiado para construir la Teoría de Situación Específica ya que permitió su desarrollo teniendo una base conceptual, teórica e indicadores empíricos con los cuales los conceptos (variables) pueden ser medidos en diversos contextos (investigación y práctica).

\begin{abstract}
Introduction: Situation-Specific Theories (SST) address a relatively concrete and specific phenomenon that allow describing, explaining and predicting how it occurs. Their main function is to provide a structure and a guidance for the interpretation of initially puzzling behaviors, processes, human responses and events. Objective: to generate a SST that explains the socio-ecological protective factors of alcohol consumption in young university students based on the analysis of McLeroy's Socio-Ecological Model (SEM). Methodology: the Conceptual-Theoretical-Empirical (C-T-E) structure proposed by Fawcett was used, which consists of five steps 1) identification of concepts, 2) classification of concepts, 3) identification and classification of propositions, 4) hierarchical order of propositions and 5) construction of the diagram. Results: the SST is the result of the C-T-E structure, contributes to the identification of a discipline-specific phenomenon, and determines concepts, relationships and possible effect of protective factors on drinking behavior. Conclusion: the method was appropriate to build the Situation-Specific Theory as it allowed its development having a conceptual and theoretical basis and empirical indicators with which the concepts (variables) can be measured in different contexts (research and practice).
\end{abstract}

\section{Palabras clave}

Consumo de bebidas alcohólicas, teoría de enfermería, universitarios, factores protectores

\section{Keywords}

Alcoholic beverage consumption, nursing theory, college students, protective factors 


\section{Introducción}

El consumo de alcohol es el factor causal de más de 200 enfermedades y trastornos, se asocia con el riesgo de desarrollar problemas de salud tales como trastornos mentales y del comportamiento, enfermedades no transmisibles como la cirrosis hepática, algunas enfermedades cardiovasculares y cerebrovasculares, así como traumatismos derivados de la violencia y los accidentes de tránsito. Dichas consecuencias se presentan con mayor frecuencia en el grupo etario de 20 a 39 años, con un 13.5 \% de las defunciones (Organización Mundial de la Salud [OMS], 2018).

En México, la población de 18 a 65 años reportó una prevalencia de consumo de alcohol excesivo explosivo en el último mes en los años 2011 a 2016, donde se observa un incremento de $12.3 \%$ a 19.8\% respectivamente; en particular en los hombres aumentó de $20.9 \%$ a $29.9 \%$ y en las mujeres de $4.1 \%$ a $10.3 \%$ en mismo periodo. Aunado a esto, el consumo de alcohol se acentúa en los jóvenes de entre 18 a 25 años donde existe una prevalencia de consumo de alcohol durante el último mes (22.1\%), sobre todo en la población universitaria donde su consumo generalmente se realiza los fines de semana, con lo cual se incrementa el riesgo de accidentes de tránsito, lesiones y homicidios no intencionales (Instituto Nacional de Psiquiatría Ramón de la Fuente [INPRFM] Instituto Nacional de Salud Pública [INSP] y Secretaria de Salud [SSA], 2017; Valdez, Pech \& Lira, 2018).

El consumo de alcohol en jóvenes tiene múltiples implicaciones sociales, psicológicas y personales por ello se llevan a cabo en los sistemas de salud grandes esfuerzos en la identificación de los factores que prevengan este consumo. Los factores protectores se pueden definir como "un atributo o característica individual, condición situacional y/o contexto ambiental que inhibe, reduce o atenúa la probabilidad del uso y/o abuso de alcohol” (Becoña et al., 2014; Callisaya, 2018). Dichos factores en el ámbito de la promoción de la salud, se han estudiado en los últimos años y se enfocan en analizar el impacto o efecto de estos factores en la conducta de consumo de algunas sustancias como el alcohol (Sainz et al., 2020). De estos estudios según la literatura localizada, son escasos lo que se han realizado bajo un modelo teórico que considere los factores protectores dentro de un contexto más amplio, con una visión ecológica y holística, que contribuya a explicar y predecir el peso de cada factor dentro de la conducta de consumo de alcohol.

Otra limitante es que la mayor parte de la bibliografía existente versa sobre los factores de riesgo, que determinan este tipo de consumo de alcohol en jóvenes y es escasa la evidencia científica referente a los factores protectores, tanto en México como en países de América Latina (Ahumada, Gámez \& Valdez, 2017; Callisaya, 2018; Mosqueda-Díaz \& Carvalho, 2011; Logan, Kilmer \& Marlatt, 2010). Es por esto, que la identificación de los factores protectores desde la percepción y experiencia de los jóvenes, es una de las tareas pendientes para poder comprender el fenómeno del consumo de drogas como el alcohol y poder intervenir a futuro de forma oportuna limitando este consumo, según las recomendaciones de la Organización de los Estados Americanos (OEA, 2019; Callisaya, 2018).

Los jóvenes universitarios como individuos se ubican en diversos contextos, son influidos y a su vez influyen a estos. De esta forma establecen manifestaciones identitarias socioculturales de su grupo, resultado de los procesos formativos en diversos lugares y se pueden perfilar por su concepción del mundo y de sí mismos que se transforma, aunando a que empiezan a rechazar el control de sus padres. Así mismo, en algunos casos abandonan sus hogares por motivos de ubicación geográfica de las universidades, teniendo la necesidad de trasladarse a ciudades más grandes y a iniciar su vida adulta con libertad y autonomía para tomar decisiones sobre sí mismos y sobre sus comportamientos (Suárez-Zozaya, 2018; Monteiro, Tavares \& Pereira, 2018; Reynaga-Ornelas et al., 2015). La conducta puede tener determinantes que la influyen como las expectativas para el consumo de alcohol. Algunas expectativas de los jóvenes para consumir la sustancia son: el sentir relajación del estrés por los estudios universitarios, la perdida de la noción del tiempo, asumir y experimentar prácticas que pueden ser de riesgo, olvidar problemas personales, familiares y académicos o para socialización, observando que el consumo de alcohol en esta población es diferente desde un consumo de bajo riesgo hasta un consumo excesivo explosivo (Aguilar-Mena et al., 2019; Valdez, Pech \& Lira, 2018; OEA, 2019). Es por esto, la necesidad de indagar este tipo de consumo en este grupo que se observa estar en aumento y determinar sus probables factores protectores.

El consumo de alcohol se ha explicado a partir de los contextos donde vive y se relaciona el ser humano tales como 
la familia, la escuela, la comunidad e instituciones sociales. No obstante, generalmente se analiza este fenómeno desde una sola visión y no de manera integrativa. Siendo así, es necesario una perspectiva teórica más amplia como el Modelo Socio Ecológico (MSE) de McLeroy et al. (1988) que parte de una visión integral ecológica (Bronfenbrenner, 1979), donde la persona es parte de los cinco niveles de influencia (intrapersonal, interpersonal, institucional, comunitario y políticas públicas) de la conducta los que se influyen mutuamente, razón por la que se deberían indagar de manera conjunta; además, el MSE considera un enfoque de protección.

Este enfoque integral permite profundizar en el conocimiento de los factores protectores del consumo de alcohol, así como determinar el peso de cada uno de estos en la limitación o evitación de la conducta del consumo de alcohol, las posibles interacciones entre ellos y analizar su influencia a lo largo del continum del consumo de alcohol, que se inicia en abstinencia hasta convertirse en consumo experimental, de bajo riesgo, dependiente o de riesgo, perjudicial y excesivo explosivo (Anderson, Gual \& Colon, 2008; OMS, 2014; OMS, 2018). En base a este MSE, se puede explicar el consumo de alcohol, al considerarlo como un fenómeno integral que se determina por el individuo, la familia, comunidad, instituciones y sistema político en un marco definido por estos contextos (Pons \& Buelga, 2011).

Desde esta perspectiva McLeroy y colaboradores (1988), en su MSE categorizan los determinantes de la conducta en cinco niveles de influencia específicos para el comportamiento de la salud: nivel intrapersonal (actitudes, autoconcepto, habilidades, edad, sexo, escolaridad, resiliencia, satisfacción con la vida y fortalezas humanas); nivel interpersonal (redes sociales formales e informales, sistema de apoyo social, redes de amistad, presión de grupo, apego a los padres y a los pares (compañeros y amigos), normas sociales y apoyo social en redes sociales virtuales); nivel institucional (Instituciones sociales, dinámica familiar y clima social escolar); nivel comunitario (redes informales, iglesias, vecindarios, entorno de barrio y densidad y proximidad de los espacios de venta de alcohol); y nivel de políticas públicas (políticas públicas, procedimientos, leyes reguladoras, percepción de políticas públicas) (Falco et al., 2013; Golden \& Earp, 2012; McLeroy et al, 1988).

Este MSE es una teoría con un origen interdisciplinario que es útil para las ciencias humanísticas, y al ser utilizado para generar una nueva teoría en la disciplina de enfermería se puede considerar como una teoría prestada. Esto es de utilidad cuando los conceptos o teorías existentes no son suficientes para explicar un determinado fenómeno o explicarlo desde otra perspectiva (Walker \& Avant, 2019). Para este fin se toman prestados modelos o teorías de otros campos de estudio, como en este caso el MSE, que además se considera interdisciplinario porque permite la integración de diversas disciplinas como enfermería.

Es por esto, que surge la necesidad de crear teorías específicas para este fenómeno, que de acuerdo con Fawcett (2006, p19) y Meleis (2012), se les denomina Teorías de Situación Específica (TSE) porque abordan un fenómeno relativamente concreto y específico al describir qué es el fenómeno, explicar por qué ocurre o predecir cómo ocurre, siendo su principal función proporcionar una estructura para la interpretación de comportamientos, situaciones, procesos, respuestas y eventos.

Por lo anterior, el objetivo del presente estudio es generar una TSE que explique los factores protectores socio ecológicos del consumo de alcohol en jóvenes universitarios, producto del análisis del Modelo Socio Ecológico (MSE) de McLeroy y colaboradores (1988).

\section{Método}

Es un estudio metodológico, utilizando el método de Estructura Conceptual-Teórico-Empírico (C-T-E), el cual es utilizado para analizar los componentes estructurales y de contenido de una teoría, para determinar lo que indica un Modelo Conceptual, Teoría de Rango Medio o una Teoría de Situación Específica; la estructura C-T-E permite la identificación de los conceptos y las proposiciones que establece, así como la identificación de un método empírico para la investigación. El resultado de la estructura C-T-E es una versión clara, concisa y gráfica de la estructura de los componentes de la teoría; esta técnica está compuesta por cinco pasos:1) la identificación de conceptos, 2) la clasificación de los conceptos, 3) la identificación y clasificación de las proposiciones 4) el orden jerárquico de proposiciones y 5) la construcción del diagrama (Fawcett, 1999). 
El periodo de elaboración y análisis fue de noviembre de 2019 a enero 2021. Como referente teórico se utilizó el Modelo Socio Ecológico (MSE) de McLeroy (1988), el cual se desprende del Modelo Ecológico de Broffenbrenner (1979) y considera los trabajos de Belsky (1980). Este modelo centra la atención en factores ambientales individuales y sociales como objetivos para las intervenciones de promoción de la salud.

\section{Resultados}

El resultado principal de la Substracción es la Estructura Conceptual Teórica Empírica (C-T-E), que es una versión clara, concisa y gráfica de la estructura de los componentes de la teoría y su contenido, así como de sus indicadores empíricos, en su conjunto, permiten el diseño de la TSE de factores protectores socio ecológicos del consumo de alcohol en jóvenes universitarios.

\section{Paso 1: Identificación de conceptos.}

El primer paso en la estructura C-T-E es la identificación de los conceptos (Fawcett, 1999); para el presente estudio en primer lugar se seleccionó el Modelo Socio Ecológico (MSE) de McLeroy et al., (1988). El MSE está compuesto por cinco grandes conceptos (niveles) que se consideran y son parte de este sistema y son los niveles: intrapersonal, interpersonal, institucional, comunitario y de políticas públicas, donde el principio de este modelo es que el contexto ambiental interactúa con el individuo y lo influye para desarrollar conductas saludables o de riesgo.

Debido a que este modelo permite la identificación de los diversos elementos del medio ambiente que interactúan con el individuo, con el propósito de promover las conductas saludables como el no consumo de alcohol. Por este motivo, en cada uno de estos factores, de acuerdo con la revisión de la literatura, se consideraron diversos conceptos (variables) que puedan contribuir a explicar los factores protectores en cada uno de dichos niveles.

El nivel intrapersonal está constituido por las características físicas, biológicas y psicológicas del individuo que influyen en la conducta (McLeroy et al., 1988) e incluye factores como la edad, el sexo, la escolaridad, la resiliencia, la satisfacción con la vida y las fortalezas humanas, es decir, son características intrínsecas del individuo; en el nivel interpersonal se consideran todos aquellos grupos, personas, regulaciones con las que el individuo está en contacto, se relaciona e interactúa como las redes sociales próximas al joven universitario, escenarios en los que interactúa el individuo en desarrollo desde el nacimiento. Se identificaron como factores el apego a los padres y a los pares (compañeros y amigos), las normas sociales y el apoyo social en redes sociales virtuales.

El nivel institucional se refiere a las instituciones sociales con características de organización, con reglas y regulaciones formales e informales, con las que el individuo tiene contacto, las influye y se ve influido por ellas; de acuerdo a la revisión de la literatura se identificaron como factores a la dinámica familiar y el clima social escolar. El nivel comunitario se refiere a las relaciones entre las organizaciones y grupos en un área definida los cuales influyen en las normas, valores y comportamientos de los individuos que la integran. En este nivel se identificaron como factores el entorno de barrio y la densidad y proximidad de los espacios de venta de alcohol. Por último, el nivel de políticas públicas se refiere al uso de políticas, procedimientos y leyes reguladoras para proteger la salud de la comunidad, que además pueden incentivar conductas positivas o restringir comportamientos negativos. En este nivel se incluye como factor a la percepción de políticas públicas; y la conducta resultado de esta teoría es el consumo de alcohol (abstinencia, consumo experimental, de bajo riesgo, dependiente, perjudicial y excesivo explosivo de alcohol).

\section{Paso 2: Clasificación de los conceptos.}

El segundo paso de la estructura C-T-E es la clasificación de los conceptos de la Teoría (Fawcett, 1999). Los conceptos se clasifican de acuerdo con el continuum de observabilidad de Kaplan (1964), que indica qué tan directamente observable es un fenómeno. Por ello, se identifican las principales variables del estudio, se analizan los niveles de abstracción, se identifican las relaciones hipotéticas entre las variables y conectan las bases teóricas con los aspectos 
operacionales del estudio (Dulock \& Holzemer, 1991).

De acuerdo con la perspectiva ecológica, se considera que el consumo de alcohol es multifactorial y que los factores incluidos en los niveles intrapersonal, interpersonal, institucional, comunitario y de políticas públicas pueden influir o inhibir el consumo de alcohol (Sánchez-Sosa et al., 2014).

Dichos factores del MSE tienen un nivel de abstracción alto por lo cual no pueden ser medidos directamente en la realidad. Para el desarrollo de la Teoría de Situación Específica se conservan estos conceptos considerándolos como niveles y en base a la evidencia empírica consultada, se definieron los factores en cada nivel, tomando en cuenta la conducta o variable resultado que es el consumo de alcohol. Así mismo, se conceptualizaron los elementos estructurales de cada uno de los factores y los instrumentos de medición como se presentan en la tabla 1.

Tabla 1. Instrumentos de medición de las variables de estudio

\begin{tabular}{|c|c|c|}
\hline Nivel & Concepto o factor & Instrumento de medición \\
\hline \multirow{3}{*}{ Intrapersonal } & Resiliencia & $\begin{array}{l}\text { Escala de Resiliencia (ER) de Wagnil y Young (1993), validado al espa- } \\
\text { ñol por Heilemann, Lee y Kury (2003) y aplicado en México por López } \\
\text { et al., 2015) }\end{array}$ \\
\hline & Satisfacción con la vida & $\begin{array}{l}\text { Escala de Satisfacción con la Vida (SWLS) por sus siglas en inglés de } \\
\text { Diener et al., 1985. Validado al español por Atieza et al., } 2000 \text { y aplica- } \\
\text { da en México por Padrós, Gutiérrez y Medina (2015) }\end{array}$ \\
\hline & Fortalezas Humanas & $\begin{array}{l}\text { Test breve de Fortalezas Humanas (VIA-IS) de Peterson y Seligman } \\
\text { (2004), adaptado al español por Cosentino y Castro-Solano (2008) }\end{array}$ \\
\hline \multirow{2}{*}{ Interpersonal } & $\begin{array}{l}\text { Apego a los padres y pa- } \\
\text { res }\end{array}$ & $\begin{array}{l}\text { Inventario de Apego a los Padres y Pares (IPPA) por sus siglas en inglés, } \\
\text { de Armsden y Greenberg (1987), validada al español por Pardo et al., } \\
2006\end{array}$ \\
\hline & $\begin{array}{l}\text { Apoyo Social en Redes so- } \\
\text { ciales virtuales }\end{array}$ & $\begin{array}{l}\text { Escala de Apoyo Social a través de Redes Sociales (ASPRS), diseñado } \\
\text { para población mexicana por González, Landero y Días (2013), aplica- } \\
\text { do en México por Navarro (2016). }\end{array}$ \\
\hline \multirow[b]{2}{*}{ Institucional } & Dinámica familiar & $\begin{array}{l}\text { Apgar Familiar de Smilkstein (1978), validada al español por Bellon et } \\
\text { al., } 1996 \text { y aplicado en México por Gómez y Ponce (2010). }\end{array}$ \\
\hline & Clima social Escolar & $\begin{array}{l}\text { Escala de Seguridad y Clima Escolar de California (CSCS, por sus siglas } \\
\text { en inglés) de Furlong, Morrison y Boles (1991), Adaptada al español } \\
\text { por Trianes et al., } 2006\end{array}$ \\
\hline Políticas Públicas & $\begin{array}{l}\text { Percepción de políticas } \\
\text { publicas }\end{array}$ & $\begin{array}{l}\text { Instrumento de Apoyo a las Políticas Públicas para reducir los Proble- } \\
\text { mas Asociados con el Alcohol (APRPAA), elaborado por el Instituto } \\
\text { Australiano de Salud y Bienestar (AIHW, 2010), adaptado para inves- } \\
\text { tigación por Buykx et al., 2016, traducido y adaptado al español por el } \\
\text { autor de este estudio }\end{array}$ \\
\hline Conducta Resultado & Consumo de alcohol & $\begin{array}{l}\text { Instrumento AUDIT, de Babor et al., (1989) adaptado para la población } \\
\text { mexicana por De la Fuente y Kershenobich (1992), }\end{array}$ \\
\hline
\end{tabular}




\section{Paso 3: Identificación y clasificación de las proposiciones.}

Las proposiciones son enunciados declarativos sobre uno o más conceptos de la teoría. El MSE no presenta de manera explícita supuestos teóricos, sin embargo, del contenido de la descripción del modelo se extrajo una proposición teórica principal:

1) El comportamiento o conducta del individuo está determinada por los niveles intrapersonal, interpersonal, institucional, comunitario y de políticas públicas.

En otras palabras, los autores indican que "las conductas específicas de salud, como en este caso el consumo de alcohol, tienen múltiples influencias a nivel intrapersonal, interpersonal, institucional, comunitario y de políticas públicas". Las influencias relacionadas con las conductas de salud determinan en este caso la conducta. Este enfoque ecológico debe ser específico al comportamiento a estudiar, centrándose en las posibles influencias más relevantes en cada nivel.

El MSE no indica otras proposiciones explicitas entre los niveles y la conducta, por lo que, en base a la proposición anterior y en la evidencia empírica consultada, se describen las relaciones entre los factores incluidos en cada nivel de la TSE los cuales influyen en la variable resultado "El consumo de alcohol" y teniendo en cuenta el enfoque de factores protectores se describen las siguientes proposiciones:

1. La edad, el sexo, la escolaridad, la resiliencia, la satisfacción con la vida y las fortalezas humanas influyen en el consumo de alcohol.

2. El apego a los padres y pares, las normas sociales y el apoyo social en redes sociales virtuales influyen en el consumo de alcohol.

3. La dinámica familiar y el clima social escolar determinan en el consumo de alcohol.

4. El entorno de barrio y la densidad y proximidad de los espacios de venta de alcohol influyen en el consumo de alcohol.

5. La percepción de políticas públicas determina el consumo de alcohol.

6. Los niveles intrapersonal, interpersonal, institucional, comunitario y de políticas públicas influyen en el consumo de alcohol.

Estas 6 proposiciones se consideran de tipo relacional, ya que indican un vínculo entre dos o más conceptos (Fawcett, 1999). En la figura 1 se muestra la representación de las proposiciones en la Teoría de Situación Específica de Factores Protectores Socio Ecológicos del Consumo de Alcohol en Jóvenes Universitarios (FAPSECA). 


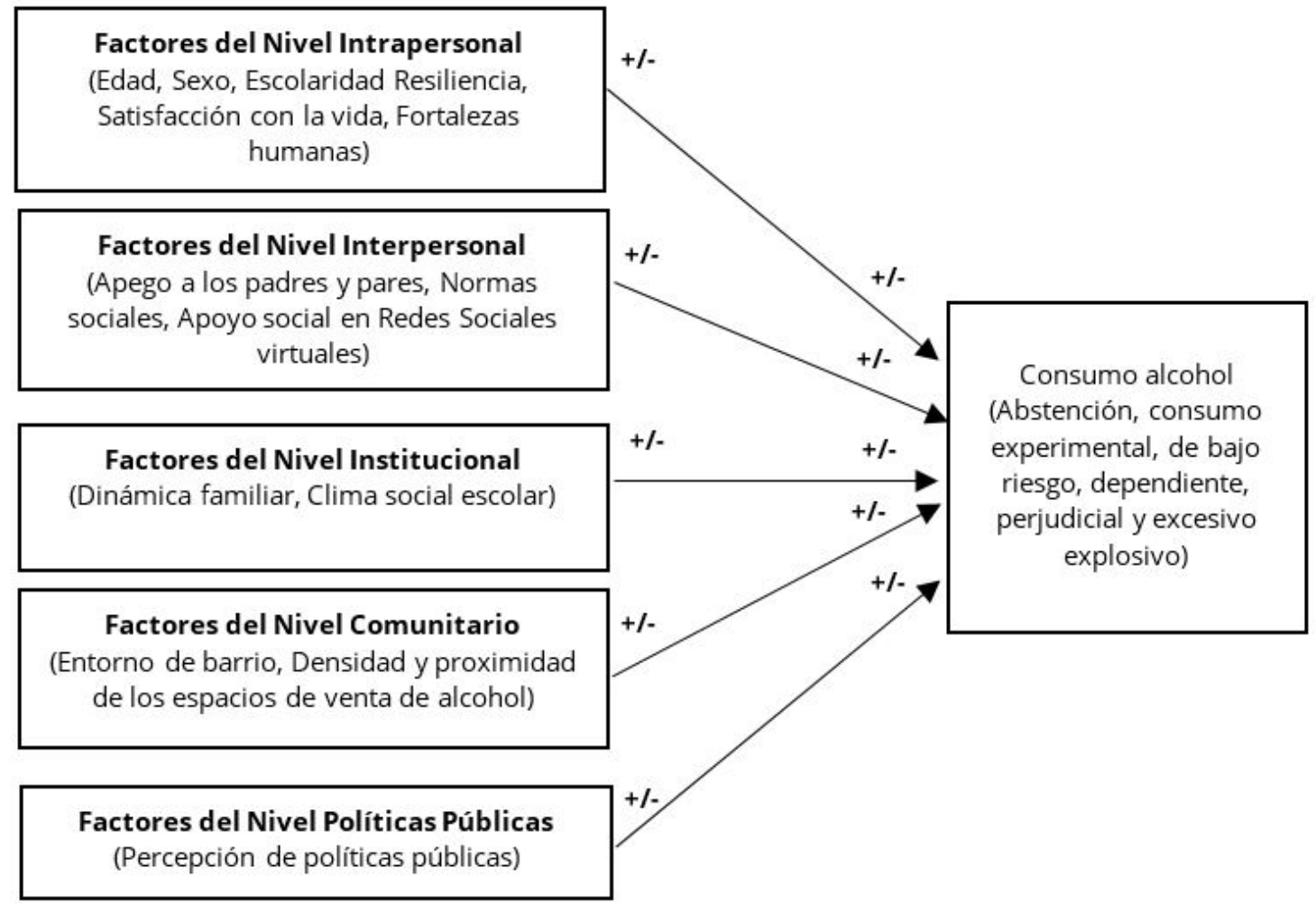

Figura 1. Teoría de Situación Específica: Factores Protectores Socio Ecológicos del Consumo de Alcohol en Jóvenes Universitarios (FAPSECA).

\section{Paso 4: El orden jerárquico de las proposiciones.}

El cuarto paso consiste en el ordenamiento de las proposiciones en conjuntos. Estas pueden ser ordenadas jerárquicamente de acuerdo con su nivel de abstracción, por razonamiento inductivo o deductivo; para el presente estudio las proposiciones se clasificaron de acuerdo con su nivel de abstracción (Fawcett, 1999). De esta forma, la principal y única proposición del Modelo Socio Ecológico de McLeroy et al. (1988) es muy amplia y general, la cual se considera abstracta, ya que es aplicable a diversos fenómenos generales. Por lo tanto, las seis proposiciones planteadas en la Teoría de Situación Específica de este estudio se consideran proposiciones medianamente abstractas, porque se enfocan a un fenómeno específico. Por último, las proposiciones conformadas por las variables serán operacionalizadas (hipótesis de estudio), se consideran concretas, porque se relacionan con los indicadores empíricos del estudio.

\section{Paso 5: La construcción del diagrama.}

El último paso es la construcción del diagrama de la Estructura C-T- E (Figura 2). En este se observan las relaciones en los diferentes niveles: en el Conceptual se puede observar los conceptos o niveles del MSE de McLeroy (1988), que se usaron para guiar esta estructura; en el nivel Teórico, se presentan los conceptos o factores que integran la TSE y que están agrupados en cada uno de los niveles de influencia; y en el nivel empírico se observan los instrumentos de evaluación que se utilizarán para medir cada uno de los conceptos (variables) de la TSE.

Como parte de la Estructura Conceptual-Teórico-Empírica (C-T-E) se establecieron las nuevas definiciones conceptuales a partir de los conceptos de la teoría madre (Modelo socio ecológico de McLeroy, 1988), así como la revisión de la literatura para contribuir a establecer las nuevas definiciones conceptuales con base en la evidencia empírica. 
Figura 2. Estructura Conceptual Teórico Empírico (C-T-E)

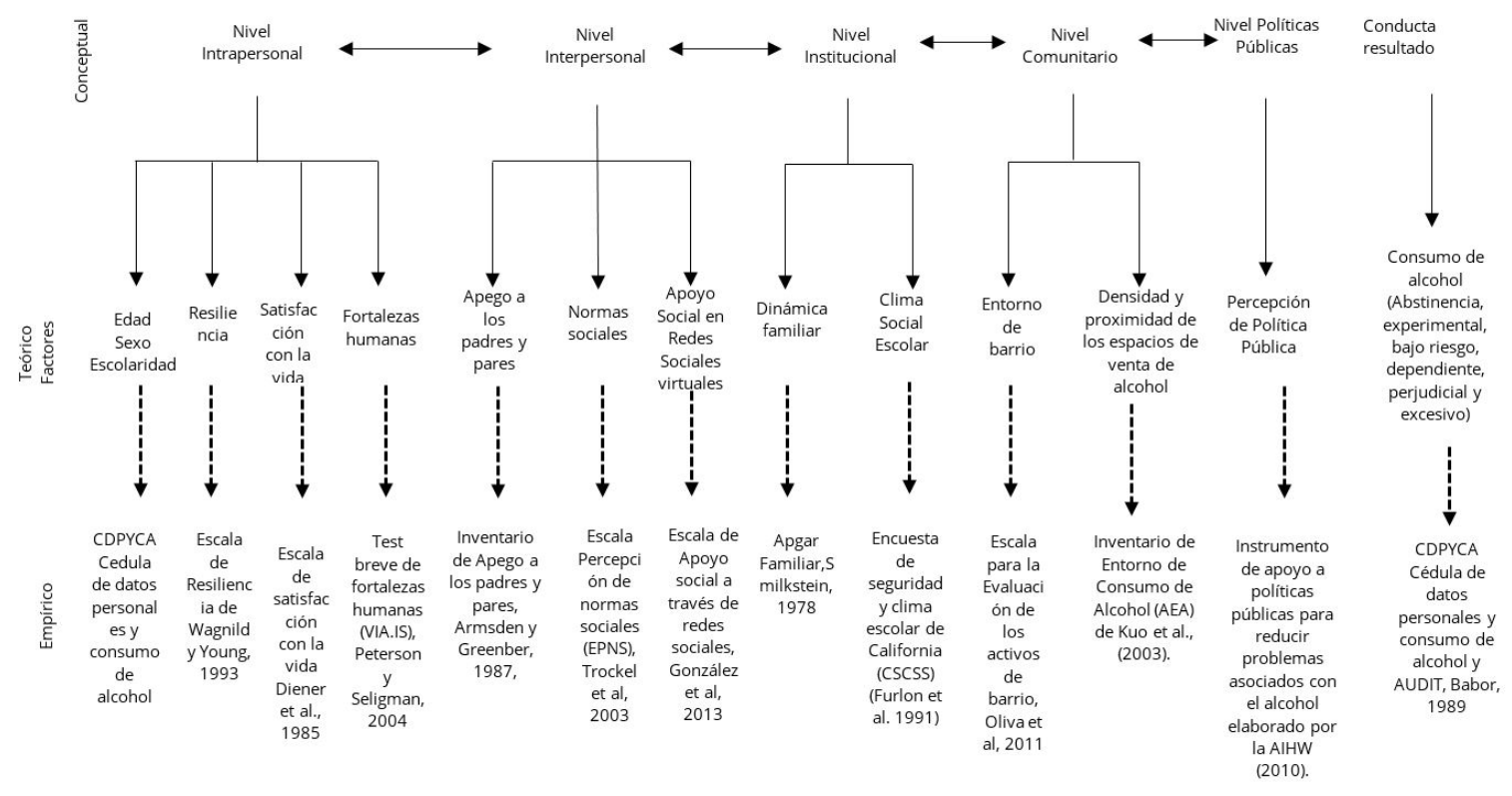

A continuación, se presentan las nuevas definiciones conceptuales de los factores protectores:

Edad: corresponde al número de años cumplidos. Se ha observado que la edad puede ser un factor protector o de riesgo (Armendáriz et al., 2012; Morera et al., 2015; Tavolacci et al., 2016), además se han encontrado que la población de 18 a 24 años es quienes reportan un mayor número de episodios de consumo de alcohol (OPS, 2015; ECONDAT, 2017; OMS, 2018; OEA, 2019). Así mismo, cuando incrementa la edad, los patrones de consumo pueden cambiar en la medida que se relacionan con otros factores como el nivel socioeconómico, el ambiente escolar, la carrera o licenciatura que estudian y la independencia familiar y económica (Armendáriz et al., 2012; Barradas, Fernández, \& Gutiérrez, 2016; Moure-Rodríguez et al., 2016; OMS, 2018).

Sexo: son las diferencias biológicas para distinguir entre hombre y mujer. Se ha observado que el sexo femenino parece ser un factor de protección porque se ha presentado en las jóvenes universitarias menores prevalencias de consumo de alcohol en comparación de los hombres, quienes han presentado un mayor riesgo de beber en exceso, quienes en su mayoría tuvieron un consumo experimental en edades tempranas (12 a 14 años) desarrollando más tarde un consumo más frecuente y elevado en cantidad de ingesta de bebidas alcohólicas (Ahumada, Gámez \& Valdez, 2017; Barradas, Fernández, \& Gutiérrez, 2016; Fernández \& Corral, 2018; García-Carreto, 2019; Reckdenwald et al., 2016; Sharma, et al., 2018; Morera et al., 2015; Moure-Rodríguez et al., 2016; OMS, 2018).

Escolaridad: se refiere al número de años de estudios formales que reporta el joven universitario, además, se considera el grado o semestre y la carrera universitaria que actualmente cursa. Se ha documentado que a menor escolaridad se observa mayor probabilidad de desarrollar la conducta de consumo de alcohol (Villacé, Fernández \& Da Costa Júnior, 2013; Mantilla-Toloza, Villamizar \& Peltzer, 2016), por lo que podría deducirse que el tener una mayor escolaridad podría ser un factor protector de esta conducta. Así mismo, un elemento relevante de la escolaridad es la carrera que estudian los jóvenes universitarios, pues cada área de estudios proporciona diferentes ambientes escolares que pueden ayudar a limitar o favorecer ciertas conductas como el consumo de alcohol. Se ha documentado que algunas áreas como las ciencias exactas, ingenierías o artes son los que han reportado mayores prevalencias de consumo de alcohol, en comparación con el área de la salud y humanidades (Armendáriz, 2012; Barradas, Fernández, \& Gutiérrez, 2016; Mantilla-Toloza et al., 2016). Sin embargo, se debe resaltar que la carrera puede tener un efecto protector o de riesgo, esto depende de la interacción que tenga con otros factores de los cinco niveles de influencia analizados en este estudio (intrapersonal, interpersonal, institucional, comunitario, políticas públicas). 
Resiliencia: es la percepción sobre la capacidad que tienen los jóvenes universitarios para superar los eventos adversos de la vida y le permite ser capaz de obtener una adaptación positiva ante alguna situación negativa y mantener dicha adaptación para evitar conductas de riesgo como el consumo de alcohol. Se ha identificado que la resiliencia es uno los factores protectores más estudiados en el ámbito de las adicciones (Barcelata \& Rivas, 2016; García, García \& Rivera, 2015; Trigueros et al., 2017). Se ha documentado que, a mayor puntaje o nivel de resiliencia, menor es el consumo de alcohol en los adultos jóvenes y jóvenes universitarios (López et al., 2015; Reyes et al., 2018; Sánchez, 2018). Así mismo, este factor contribuye a superar los efectos negativos de esta conducta y de la misma manera puede ayudar a evitar el consumo de alcohol en los jóvenes universitarios (García-Cortés et al., 2015; García del Castillo et al., 2016; López et al., 2015; Reyes et al., 2018; Sánchez, 2018).

Satisfacción con la vida: se considera como un juicio global sobre las condiciones de vida actual, la consecución o logro de lo que se considera importante para la vida del joven universitario y lo compara con un estándar o criterio subjetivo, como analizar sus logros actuales con respecto a sus expectativas. Se ha identificado que este factor se encuentra presente en una mayor proporción en las mujeres jóvenes (Yi et al., 2017), cuando esta satisfacción es baja hay mayor probabilidad de que presenten un aumento en el consumo de alcohol, por lo que se infiere que a mayor satisfacción con la vida menor será el consumo de alcohol. Además, se ha observado que la falta de satisfacción con la vida puede influir en el consumo de otras sustancias (Lew et al., 2019; Mason-Jones \& Cabieses, 2015; Mies et al., 2018; Peltzer \& Pengpid, 2015).

Fortalezas humanas: se refiere a la percepción sobre características psicológicas positivas del joven universitario como el optimismo, la esperanza, la honestidad, la perseverancia, la capacidad de fluir y la competencia percibida, que alientan al joven universitario a alcanzar una virtud o una conducta saludable como el no consumo de alcohol. Este factor se ha destacado como un posible protector ante el consumo de alcohol en los adolescentes y jóvenes, ya que se ha relacionado positivamente con conductas saludables como el no consumo de alcohol (Barrantes-Brais, Ureña \& Gutiérrez, 2015). Además, se ha identificado que cuando hay una mayor presencia de fortalezas en los jóvenes tienen un menor involucramiento con conductas que puedan perjudicar su salud (Andrade et al., 2017; Barrantes-Brais et al., 2015; Logan, Kilmer \& Marlatt, 2010; Ovejero \& Cardenal, 2015).

Apego a los padres y pares: corresponde a la percepción del joven universitario sobre el vínculo afectivo el cual es perdurable en el tiempo y tiene como objetivo el mantenimiento y búsqueda de la cercanía entre los individuos con una figura central (padres) o varias figuras afectivamente cercanas a él (amigos, compañeros), lo que genera seguridad en sí mismo. Este apego puede ser un factor protector que limita el consumo de alcohol, en adolescentes y jóvenes universitarios (Arunachalam \& Nguyen, 2016; Moreno-Carmona \& Palomar-Lever, 2017; Simons-Morton et al., 2016). Se ha observado que cuando los padres tienen un estilo parenteral negligente, ser padres ausentes y estar separados se puede incrementar la probabilidad de consumo de alcohol en los hijos sobre todo cuando son adolescentes y jóvenes (Minaie et al., 2015). Otros autores (Moore et al., 2018; Minaie et al., 2015; Moreno-Carmona \& Palomar-Lever, 2017; McKay, 2015) señalan que el apego a los padres puede incrementar en los jóvenes el bienestar y ser factor protector de conductas de riesgo como el consumo de alcohol. Del mismo modo el apego a los pares (amigos) puede ser un factor protector, cuando la influencia que ejercen estos sea para generar conductas saludables, pues se ha reportado que cuando los pares no consumen alcohol, existe una mayor probabilidad de que los jóvenes no desarrollen esta conducta (Yuksek \& Solakoglu, 2016; Simons-Morton et al., 2016; Yurasek et al., 2019).

Normas sociales: se consideran como un conjunto de enunciados o disposiciones y reglas que pueden orientar la conducta de consumo de alcohol de los jóvenes universitarios y se perciben en función del consumo o no consumo de los amigos y familiares cercanos. Estas se consideran como descriptivas (percepción del comportamiento personal sobre las conductas de consumo de alcohol en relación a la cantidad y frecuencia de consumo en comparación con sus amigos y/o compañeros de su escuela, facultad, universidad o lugar de trabajo) y normas prescriptivas (percepción de la aprobación o desaprobación de su propio consumo de alcohol por parte de sus amigos o compañeros). Este factor se puede considerar como un predictor de la conducta del consumo de alcohol porque si el joven universitario percibe que esta conducta es de aceptación por su entorno, se incrementa la probabilidad de consumo (Alonso, et al., 2009; Massengale et al., 2017). Así mismo, se ha identificado que cuando el joven universitario percibe la aceptación de esta conducta por sus compañeros más cercanos, ésta puede relacionarse significativamente con la conducta del consumo de alcohol (Alonso et al., 2019) ya que la identidad social y la aprobación percibida 
pueden ser predictores del consumo alcohol (Gabbiadini et al., 2017; Massengale et al., 2017). Sin embargo, se infiere que las normas sociales pueden actuar en forma positiva en los jóvenes universitarios para disminuir el consumo o incrementar la probabilidad de no consumir alcohol (Scoppetta et al., 2019).

Apoyo social en redes sociales virtuales: es la percepción de los jóvenes universitarios de contar con ayuda emocional, cognitiva e instrumental a través de las redes sociales. En la actualidad las redes sociales virtuales tienen una influencia significativa en los jóvenes, dado que la percepción del apoyo que pueden recibir por este medio virtual es comparable con la percepción de apoyo recibido de manera presencial (Ortiz-Peña et al., 2019; González-Ramírez, Landero-Hernández \& Díaz-Rodríguez, 2013). Se ha identificado que este apoyo favorece los estados de bienestar, además de percibir como efectiva la ayuda de amigos cercanos para resolver problemas (Navarro-Oliva et al., 2020), siendo este factor considerado como protector porque es útil para la promoción de la salud y para disminuir o prevenir el consumo de alcohol en los jóvenes universitarios, por la influencia que tiene en la conducta de la generación actual (Ortiz-Peña et al., 2019; González-Ramírez, Landero-Hernández \& Díaz-Rodríguez, 2013; Navarro-Oliva et al., 2020).

Dinámica familiar: corresponde a la percepción de los jóvenes universitarios sobre el nivel de funcionamiento familiar de forma global donde se valora la adaptación, cooperación, desarrollo (maduración física y emocional), afecto y la capacidad resolutiva, características que favorecen un ambiente familiar positivo (Smilksteing, 1978). Se ha señalado (Grevenstein, Nagy \& Jungaberle, 2015; Sudhinaraset, Wigglesworth \& Takeuchi, 2016) que la dinámica familiar en los jóvenes universitarios desempeña un rol protector significativo, sobre todo en aquellos jóvenes que siguen viviendo con sus padres durante esta etapa. Así mismo, Alonso et al. (2017) indicaron que cuando disminuye esta dinámica familiar, puede favorecer el desarrollo de la conducta de consumo de alcohol. Además, se ha identificado que cuando los jóvenes viven con un solo progenitor o con otros miembros de la familia que no sean sus padres, hay una mayor probabilidad del consumo de alcohol (Uroz-Olivares et al., 2018). Por el contrario, cuando esta dinámica familiar se percibe como positiva y hay una mayor comunicación al interior de la familia, esto puede generar en los jóvenes, conductas saludables como el no consumo de alcohol (García-Cortés et al., 2015; Musitu-Ochoa et al., 2015).

Clima social escolar: se refiere a la percepción que los jóvenes universitarios tienen sobre la institución escolar, respecto al contexto en el cual desarrollan sus actividades académicas habituales, el cual favorece conductas saludables. La universidad es el lugar donde transcurre la mayor parte de la vida de los jóvenes (González-Bello, 2018; Tomzyk et al., 2015). Así, el clima que se desarrolle y se perciba en estas instituciones puede ser un factor protector, en la medida en que evite o limite el inicio temprano de conductas nocivas como el consumo de alcohol y promueva conductas saludables (Bacio et al., 2015). Este clima se puede desarrollar de la misma manera en los entornos virtuales, en los cuales el estudiante toma sus clases e interactúa con sus compañeros y profesores, recibiendo así de alguna forma servicios escolares, recursos tecnológicos y de simulación (Cedeño \& Murillo, 2020; Pérez, Miguelena, \& Diallo, 2016).

Entorno de barrio: corresponde a la percepción que los jóvenes universitarios tienen sobre el vecindario donde viven con respecto al apoyo, seguridad que brinda el barrio, sentido de pertenencia, control social y las actividades que ofrecen para los jóvenes universitarios las cuales pueden promover conductas saludables. Este factor se ha identificado como protector en la medida que los integrantes del barrio o vecindario promuevan actividades saludables, de integración y vigilancia, para que los jóvenes se sientan seguros y protegidos (Oliva-Delgado et al., 2012). Por lo que una percepción positiva de este entorno limita las conductas nocivas como el consumo de alcohol (Cambrón et al., 2017; Fagan et al., 2015; Oliva-Delgado et al., 2012).

Densidad y proximidad de los espacios de venta de alcohol: se considera como el número de lugares de venta de bebidas alcohólicas, donde los jóvenes universitarios perciben que pueden comprar o consumir, y se clasifica como establecimientos que tienen venta y consumo de bebidas alcohólicas (bares, casinos, discoteca, inmuebles construidos o habilitados para feria, restaurante-bar) y establecimientos con venta de bebidas alcohólicas (tienda de abarrotes, supermercados y tiendas de conveniencia). Se ha observado que en las localidades donde se ha reducido la densidad de los puntos de venta, se ha disminuido el consumo de alcohol en la población circundante sobre todo en los jóvenes, ya que esto reduce la probabilidad de consumo (Zhang et al., 2015; Pastor et al., 2020). 
Percepción de política pública: comprende la apreciación que tienen los jóvenes universitarios sobre las políticas públicas de regulación del consumo de alcohol como: el precio de las bebidas alcohólicas, el mercadeo y la información, así como la reducción de daños y conducir bajo los efectos del alcohol son elementos que podrían representar en su conjunto un factor protector para evitar o reducir el consumo de alcohol. Se ha considerado que las políticas públicas en materia de alcohol son eficaces y logran su objetivo en la medida en que la población y sobre todo los jóvenes las perciban como útiles y coherentes con las necesidades y circunstancias en las que viven (Casswell et al., 2016; Delamaza \& Thayer, 2016).

Consumo de alcohol: es la ingesta de bebidas con contenido alcohólico (etanol) considerada como un continuum de severidad que inicia en abstinencia, hasta convertirse en consumo experimental, de bajo riesgo, dependiente o de riesgo, perjudicial y excesivo explosivo (OMS, 2014; OMS, 2018). En la etapa universitaria algunos jóvenes inician su vida laboral, lo cual puede aumentar o propiciar el consumo de alcohol, ante la facilidad de acceso y la mayor capacidad de adquisición, aunando a la ausencia o limitada supervisión y control por parte de los padres, o en su defecto, en el núcleo familiar esta conducta es permitida y aprendida (Armendáriz-García et al, 2014; García-Carreto, 2019; Del Pino \& Correa, 2016; Uroz-Olivares et al, 2018).

\section{Discusión}

El uso del MSE tiene grandes aportes para la enfermería, pues éste es congruente con la enfermería que es holística, pues ambos consideran que la persona es un todo integrado. Por ello el individuo es visto en todos sus entornos, facetas y complejidades para poder apreciar sus relaciones, contrastes, interacciones, procesos, los que pueden coincidir con la visión de enfermería de brindar cuidados de una manera integral y total. Además, el desarrollo de teorías de enfermería es un componente esencial de la erudición enfermera, al fomentar el conocimiento para la disciplina (Alligood, 2015) y mejorar comprensión de los fenómenos actuales, con una visión cada vez más amplia y apoyadas por diversos enfoques interdisciplinarios en diversos contextos.

Las teorías de situación específica se definen como la articulación coherente de un conjunto de conceptos que describen y explican las relaciones de un fenómeno en particular y por lo tanto, describen y proporcionan marcos teóricos para tratar de explicar una conducta o comportamiento de los individuos y grupos. Así como las experiencias de las personas con los síntomas proporcionan los medios para comprender las respuestas humanas a las situaciones de salud y enfermedad, proponen nuevas formas de indagar un fenómeno de estudio entre otros (Fawcett, 2012; Meleis, 2012).

Los factores protectores del consumo de alcohol tienen como finalidad disminuir, prevenir o limitar el consumo de alcohol en los jóvenes universitarios. Estos factores dependiendo del nivel de influencia, pueden ser positivos o negativos, dependiendo del entorno y la posición del joven en este. En este sentido la Teoría de Situación Específica que se plantea en este estudio puede orientar al profesional de enfermería y de otras disciplinas para generar estrategias de promoción de la salud y diseñar intervenciones preventivas para fortalecer estos factores protectores en los cinco niveles de influencia, para que de esta forma se pueda disminuir o evitar el consumo de alcohol en esta población.

Se debe señalar que los comportamientos de riesgo, como el consumo de alcohol y otras sustancias pueden aumentar durante la etapa universitaria, debido a que el control social disminuye durante este periodo, y las personas se vuelven más autónomos para elegir comportamientos (consumir alcohol o no consumir) y estilos de vida que no están limitados por otros (Díaz \& Muñoz, 2016). Junto con la exploración de la identidad y probar varios comportamientos, los jóvenes universitarios pueden buscar los estados alterados de conciencia que son influidos por diferentes sustancias, debido a que construir una identidad estable puede ser confuso y difícil. Es por esto, la necesidad de que las instituciones educativas y de salud trabajen en forma coordinada para poder implementar programas enfocados en la prevención de estos tipos de conductas en las que se involucren diferentes factores protectores según los niveles de influencia para que los programas e intervenciones sean eficaces y los resultados obtenidos puedan perdurar en el tiempo. 


\section{Conclusión}

El desarrollo de nuevas teorías contribuye esencialmente al crecimiento y fortalecimiento de la disciplina de enfermería y de ciencias afines, provee directrices para fortalecer la práctica y la generación de nuevas intervenciones que se enfoquen en la limitación y prevención del consumo de alcohol. El Modelo Socio Ecológico de McLeroy permitió la construcción de una estructura Conceptual-Teórica-Empírica en la Teoría de Situación Específica, que incluye conceptos que pueden ser relacionales y factibles de ser medibles y aplicables para la generación de conocimiento científico.

El presente artículo muestra las definiciones propuestas por la Teoría de Situación Específica de Factores Protectores Socio Ecológicos del Consumo de alcohol. Se plantearon los factores que pueden determinar, en cada uno de los niveles de influencia en la conducta del joven universitario, con una visión integral (ecológica). Además, se reconoce al consumo de alcohol como un continuum que puede evolucionar a lo lago de la vida del joven, iniciando desde la abstinencia, hasta un consumo excesivo explosivo (OMS, 2014; OMS, 2018).

Por lo anterior, el método propuesto por Fawcett fue apropiado para construir esta Teoría de Situación Específica, ya que permitió su desarrollo teniendo una base conceptual, teórica e indicadores empíricos con los cuales los conceptos pueden ser medidos en la realidad. Estas teorías son parte importante de la estructura del conocimiento de la disciplina de enfermería, aportando una explicación de un fenómeno especifico, relacionado con el proceso de cuidado. Esta teoría aporta a la disciplina una visión o perspectiva para el abordaje del consumo de alcohol en los jóvenes universitarios desde una perspectiva de los factores protectores, ya que hasta el momento son escasas las investigaciones realizadas con este enfoque y con una visión integral dada por los diferentes niveles de influencia para la conducta.

\section{Referencias}

Aguilar-Mena, J., Lazarevich, I., Mendoza-Rodríguez, J. M., \& López-Alavez, F. (2019). Consumo de alcohol en estudiantes universitarios mexicanos del área de salud. Revista de Ciencias, 20(1-2), 5-13. Recuperado de https://www. terceridad.net/publicaciones/Uam1912-02Aguilar\%20PDF.pdf.pdf

Ahumada, J. G. C, Gámez, M. M. E., \& Valdez, M. C. (2017). El consumo de alcohol como problema de salud pública. Ra ximhai, 13(2), 13-24. Recuperado de https://www.redalyc.org/pdf/461/46154510001.pdf

Alligood, M. R. (2015). Modelos y teorías en enfermería. ( $\left.8^{\circ} \mathrm{Ed}\right)$. Elsevier Health Sciences.

Alonso, M. M., Del Bosque, J., Gómez, M. V., Rodríguez, L., Esparza, S. E., \& Alonso, B. (2009). Percepción de normas sociales y consumo de alcohol en jóvenes universitarios. Anuario de investigación en adicciones, 10(1), 40-9. Recuperado de http://www.cucs.udg.mx/avisos/anuario_investigacion_adicciones/Anuarios_PDFs/Anuario10.pdf\#page $=41$

Alonso-Castillo, M. M., Yañez-Lozano, Á., \& Armendáriz-García, N. A. (2017). Funcionalidad familiar y consumo de alcohol en adolescentes de secundaria. Health and Addictions/Salud y Drogas, 17(1), 87-96. Recuperado de http:// dx.doi.org/10.21134/haaj.v17i1.286

Anderson, P., Gual, A., \& Colon. J. (2008). Alcohol y Atención Primaria de la salud: informaciones clínicas básicas para la identificación y el manejo de riesgos y problemas. Organización Panamericana de la Salud. Washington. DC. Recuperado de https://www.who.int/substance_abuse/publications/alcohol_atencion_primaria.pdf

Andrade, P. P., Betancourt, O. D., Moreno, C. N. D. \& Alvis, R. A. (2017). Fortalezas externas desde el modelo de desarrollo positivo de los jóvenes y consumo de sustancias en una muestra de adolescentes mexicanos y colom- 
bianos. Avances en Psicología Latinoamericana, 35(3), 515-529. Recuperado de http://www.scielo.org.co/pdf/apl/ v35n3/1794-4724-apl-35-03-00515.pdf

Armendáriz, G. N. A., Villar, L. M. A., Alonso, C. M. M., Alonso, C. B. A., \& Oliva, R. N. N. (2012). Eventos estresantes y su relación con el consumo de alcohol en estudiantes universitarios. Investigación en enfermería: Imagen y Desarrollo, 14(2), 97-112. Recuperado de https://www.redalyc.org/pdf/1452/145226758007.pdf

Armendáriz-García, N. A., Alonso-Castillo, M. M., Alonso-Castillo, B. A., López-Cisneros, M. A., Rodríguez-Puente, L. A., \& Méndez-Ruiz, M. D. (2014). La familia y el consumo de alcohol en estudiantes universitarios. Ciencia y enfermería, 20(3), 109-118. Recuperado de http://dx.doi.org/10.4067/\$0717-95532014000300010

Arunachalam, D., \& Nguyen, D. Q. V. (2016). Family connectedness, school attachment, peer influence and health-compromising behaviors among young Vietnamese males. Journal of Youth Studies, 19(3), 287-304. Recuperado de https://doi.org/10.1080/13676261.2015.1067674

Bacio, G. A., Estrada, Y., Huang, S., Martínez, M., Sardinas, K., \& Prado, G. (2015). Ecodevelopmental predictors of early initiation of alcohol, tobacco, and drug use among Hispanic adolescents. Journal of school psychology, 53(3), 195-208. Recuperado de http://dx.doi.org/10.1016/j.jsp.2015.02.001

Barcelata, E.B.E., \& Rivas, M. D. J. (2016). Bienestar psicológico y satisfacción vital en adolescentes mexicanos tempranos y medios. Revista Costarricense de Psicología, 35(2), 119-137. Recuperado de http://www.redalyc.org/articulo.oa?id=476754931005

Barradas, A. M. E., Fernández, M, N., \& Gutiérrez, S. L. (2016). Prevalencia de consumo de alcohol en estudiantes universitarios. RIDE Revista Iberoamericana para la Investigación y el Desarrollo Educativo, 6(12), 491-504. Recuperado de https://www.ride.org.mx/index.php/RIDE/article/view/213/952

Barrantes-Brais, K., Ureña, P. B., \& Gutiérrez, J. C. V. (2015). Fortalezas personales y hábitos de salud en estudiantes universitarios de ciencias del movimiento humano. Revista en Ciencias del Movimiento Humano y Salud, 11(2), 2036. Recuperado de http://dx.doi.org/10.15359/mhs.11-2.2

Becoña, I. E., Fernández del Río, E., Calafat, A., \& Fernández-Hermida, J. R. (2014). Attachment and substance use in adolescence: A review of conceptual and methodological aspects. Adicciones, 26(1), 77-86. DOI:10.20882/adicciones.137

Bronfenbrenner, U. (1979). The ecology of human development. Harvard university press.

Callisaya, J. I. A. (2018). Factores de riesgo y protección ante el consumo de drogas en jóvenes del municipio de la Paz. Drugs and Addictive Behavior, 3(1), 52-74. Recuperado de https://doi.org/10.21501/24631779.2635

Cambron, C., Kosterman, R., Catalano, R. F., Guttmannova, K., \& Hawkins, J. D. (2017). Neighborhood, family, and peer factors associated with early adolescent smoking and alcohol use. Journal of youth and adolescence, $47(2)$, 369-382. DOI: 10.1007/s10964-017-0728-y

Casswell, S., Huckle, T., Wall, M., \& Parker, K. (2016). Policy-Relevant Behaviors Predict Heavier Drinking in Both on and Off Premises and Mediate the Relationship Between Heavier Alcohol Consumption and Age, Gender, and Socioeconomic Status-Analysis from the International Alcohol Control Study. Alcoholism: clinical and experimental research, 4O(2), 385-392. DOI: 10.1111/acer.12947

Cedeño, E. R., \& Murillo, J. M. (2020). Entornos virtuales de aprendizaje y su rol innovador en el proceso de enseñan- 
za. ReHuSo: Revista de Ciencias Humanísticas y Sociales, 4(1), 119-127. Recuperado de https://doi.org/10.33936/ rehuso.v4i1.2156

Del Pino, A., \& Correa, B. (2016). Alcoholismo en jóvenes universitarios de diferentes carreras. Revista Psicología Científica, 1(11), 77-97. Recuperado de https://www.psicologiacientifica.com/alcoholismo-jovenes-universitarios/

Delamaza, G., \& Thayer, L. E. (2016). Percepciones políticas y prácticas de participación como instrumento para la gobernanza de los territorios. Un análisis comparado de escalas territoriales en el macrorregión sur de Chile. Eure, 42(27), 137-158. Recuperado de https://www.redalyc.org/articulo.oa?id=19646820006

Díaz, L. P. H., \& Muñoz, A. A. S. (2016). Vulnerabilidad al consumo de alcohol, trascendencia espiritual y bienestar psicosocial: comprobación de teoría. Revista Latino-Americana de Enfermagem, 24(e2702), 1-8. DOI: 10.1590/15188345.0688.2702

Dulock, H. L., \& Holzemer, W. L. (1991). Substruction: Improving the linkage from theory to method. Nursing Science Quarterly, 4(2), 83-87.

Fagan, A. A., Wright, E. M., \& Pinchevsky, G. M. (2015). Exposure to violence, substance use, and neighborhood context. Social science research, 49, 314-326. Recuperado de http://dx.doi.org/10.1016/j.ssresearch.2014.08.015

Falco, C., Samdal, O., Estevan, I., \& Álvarez, O. (2013). Estructuración de la estrategia para organizar actividades físico-deportivas y recreativas. Iberoamericana de Psicología del ejercicio y el deporte, 8(2), 373-391. Recuperado de http://www.redalyc.org/articulo.oa?id=311128824008

Fawcett, J. (1999). Analyzing Conceptual-Theoretical-Empirical Structures for research. En Fawcett, J. (Ed), The relationship of theory and research (pp. 27-81). Philadelphia: Davis Company.

Fawcett, J. (2006) Contemporary Nursing Knowledge: Analysis and Evaluation of Nursing Models and Theories. (2 ed). Philadelphia (PA): FA Davis Company.

Fawcett, J., \& Desanto-Madeya, S. (2012). Contemporary nursing knowledge: Analysis and evaluation of nursing models and theories. Philadelphia (PA): FA Davis Company.

Fernández, A.V., \& Corral, L.I. (2018). Consumo de alcohol y género: diferencias desde la perspectiva de los profesionales de enfermería. Nure Investigación, 15(96). Recuperado de http://www.nureinvestigacion.es/OJS/index.php/ nure/article/view/1426

Gabbiadini, A., Cristini, F., Scacchi, L., \& Monaci, M. G. (2017). Testing the model of goal-directed behavior for predicting binge drinking among young people. Substance use \& misuse, 52(4), 493-506. Recuperado de http://dx.doi. org/10.1080/10826084.2016.1245335

García del Castillo, J. A., García del Castillo-López, A., López-Sánchez, C., \& Días, P. C. (2016). Conceptualización teórica de la resiliencia psicosocial y su relación con la salud. Health and Addictions/ Salud y Drogas, 16(1), 59-68. Recuperado de https://doi.org/10.21134/haaj.v16i1.263

García, J. M. C., García, M. M., \& Rivera, S. A. (2015). Potencial resiliente en familias con adolescentes que consumen y no consumen alcohol. Acta Colombiana de Psicología, 18(2), 163-172. DOI: 10.14718/ACP.2015.18.2.14

García-Carretero, M. Á., Moreno-Hierro, L., Martínez, M. R., de los Ángeles Jordán-Quintero, M., Morales-García, 
N., \& O'Ferrall-González, C. (2019). Patrones de consumo de alcohol en estudiantes universitarios de ciencias de la salud. Enfermería Clínica, 29(5), 291-296. https://doi.org/10.1016/j.enfcli.2019.01.003

García-Cortés, J. M., García-Méndez, M., \& Rivera-Aragón, S. (2015). Potencial resiliente en familias con adolescentes que consumen y no consumen alcohol. Acta Colombiana de Psicología, 18(2), 163-172. Recuperado de http:// www.dx.doi.org/10.14718/ACP.2015.18.2.14

Golden, S.D., \& Earp. J. A. L. (2012). Social Ecological approaches to individuals and their contexts: Twenty years of Health Education \& Behavior Health Promotion Interventions. Health Education \& Behavior, 39(3), 364-372. Recuperado de https://doi.org/10.1177/1090198111418634

González-Bello, E. O. (2018). Habilidades digitales en jóvenes que ingresan a la universidad: realidades para innovar en la formación universitaria. RIDE. Revista Iberoamericana para la Investigación y el Desarrollo Educativo, 8(16), 670-687. DOI: 10.23913/ride.v8i16.363

González-Ramírez, M. T., Landero-Hernández, R., \& Díaz-Rodríguez, C. L. (2013). Propiedades psicométricas de una escala para evaluar el apoyo social a través de las redes sociales. Acta de investigación psicológica, 3(3), 1238-1247. Recuperado de https://doi.org/10.1016/S2007-4719(13)70963-3

Grevenstein, D., Nagy, E., \& Jungaberle, H. K. (2015). Development of risk perception and substance use of tobacco, alcohol and cannabis among adolescents and emerging adults: evidence of directional influences. Substance Use and Misuse, 50(3), 376-386. DOI: 0.3109/10826084.2014.984847

Instituto Nacional de Psiquiatría Ramón de la Fuente Muñiz, Instituto Nacional de Salud Pública. (2017). Encuesta Nacional de Consumo de Drogas, Alcohol y Tabaco [ENCODAT] 2016-2017: Reporte de alcohol. México, D.F. Recuperado de https://drive.google.com/file/d/1rMIKaWy34GR51sEnBK2-u2q_BDK9LA0e/view

Kaplan, A. (1964). The conduct of inquiry. San Francisco. CA: Chandler.

Lew, D., Xian, H., Qian, Z., y Vaughn, M. G. (2019). Examining the relationships between life satisfaction and alcohol, tobacco and marijuana use among school-aged children. Journal of Public Health, 41(2), 346-353. DOI:10.1093/ pubmed/fdy074

Logan, D., Kilmer, J., \& Marlatt, G. (2010). The virtuous drinker: character virtues as correlates and moderators of college student drinking and consequences. Journal or American College Health, 58(4), 317-324. DOI: $10.1080 / 07448480903380326$

López, M. T. A., Morales, G. A., Gómez, A. D. L., Parra, L. V., Reyes, A. T., Castillo, F. A. M., ... \& Zarate, M. Y. T. (2015). Resiliencia, conducta de salud y conducta de consumo de drogas en estudiantes universitarios. European Journal of Child Development, Education and Psychopathology, 3(2), 55-67. DOI: 10.1989/ejpad.v3i2.29

Mantilla-Toloza, S. C., Villamizar, C. E., \& Peltzer, K. (2016). Consumo de alcohol, tabaquismo y características sociodemográficas en estudiantes universitarios. Universidad y Salud, 18(1), 7-15. Recuperado de https://revistas.udenar. edu.co/index.php/usalud/article/view/2718/pdf

Mason-Jones, A. J., y Cabieses, B. (2015). Alcohol, binge drinking and associated mental health problems in young urban Chileans. PLoS one, 10(4). DOI: 10.1371/journal.pone.0121116

Massengale, K. E., Ma, A., Rulison, K. L., Milroy, J. J., \& Wyrick, D. L. (2017). Perceived norms and alcohol use among first-year college student-athlete's different types of friends. Journal of American college health, 65(1), 32-40. DOI: 10.1080/07448481.2016.1233557 
McKay, M. T. (2015). Parental rules, parent and peer attachment, and adolescent drinking behaviors. Substance use \& misuse, 50(2), 184-188. DOI: 10.3109/10826084.2014.962053

McLeroy, K.R, Bibeau, D., Steckler, A., \& Glanz, K. (1988). An ecological perspective on health promotion programs. Health Education Quartely, 15(4), 351-377. Recuperado de https://doi.org/10.1177/109019818801500401

Meleis, A. I. (2012). Theoretical nursing development \& progress (5th ed.). Philadelphia, Wolters Kluwer

Mies, G. W., Verweij, K. J., Treur, J. L., Ligthart, L., Fedko, I. O., Hottenga, J. J., ... y Vink, J. M. (2018). Polygenic risk for alcohol consumption and its association with alcohol-related phenotypes: Do stress and life satisfaction moderate these relationships?. Drug and alcohol dependence, 183, 7-12. Recuperado de https://doi.org/10.1016/j.drugalcdep.2017.10.018

Minaie, M. G., Hui, K. K., Leung, R. K., Toumbourou, J. W., \& King, R. M. (2015). Parenting style and behavior as longitudinal predictors of adolescent alcohol use. Journal of studies on alcohol and drugs, 76(5), 671-679. Recuperado de https://doi.org/10.15288/jsad.2015.76.671

Monteiro, S., Tavares, J., \& Pereira, A. (2018). Adultez emergente: na fronteira entre a adolescência e a adultez. Revista ambiente educação, 2(1), 129-137. Recuperado de http://publicacoes.unicid.edu.br/index.php/ambienteeducacao/article/view/545/512

Moore, G. F., Cox, R., Evans, R. E., Hallingberg, B., Hawkins, J., Littlecott, H. J., ... \& Murphy, S. (2018). School, peer and family relationships and adolescent substance use, subjective wellbeing and mental health symptoms in wales: a cross sectional study. Child indicators research, 11(6), 1951-1965. Recuperado de https://doi.org/10.1007/s12187017-9524-1

Moreno-Carmona, N. D. M., \& Palomar-Lever, J. P. (2017). Factores familiares y psicosociales asociados al consumo de drogas en adolescentes. Interamerican Journal of Psychology, 51(2), 141-151. Recuperado de https://www.redalyc.org/pdf/284/28454546001.pdf

Moreno-Carmona, N. D. M., \& Palomar-Lever, J. P. (2017). Factores familiares y psicosociales asociados al consumo de drogas en adolescentes. Interamerican Journal of Psychology, 51(2), 141-151. Recuperado de https://www.redalyc.org/pdf/284/28454546001.pdf

Morera, J. A. C., Noh, S., Hamilton, H., Brands, B., Gastaldo, D., \& Wright, M. D. G. M. (2015). Sociocultural factors e drug consumption among costarrican university students. Texto \& Contexto - Enfermagem, 24(spe), 145-153. Recuperado de https://dx.doi.org/10.1590/0104-07072015001170014

Mosqueda-Díaz, A., \& Carvalho, M. G. F. (2011). Factores protectores y de riesgo familiar relacionados al fenómeno de drogas, presentes en familias de adolescentes tempranos de Valparaíso, Chile. Rev. Latino Americana Enfermagem, 19(esp), 789-795. Recuperado de http://www.scielo.br/pdf/rlae/v19nspe/17.pdf

Moure-Rodríguez, L., Doallo, S., Juan-Salvadores, P., Corral, M., Cadaveira, F., \& Caamaño-Isorna, F. (2016). Consumo intensivo de alcohol y cannabis, y prácticas sexuales de riesgo en estudiantes universitarios. Gaceta Sanitaria, 30(6), 438-443. Recuperado de http://dx.doi.org/10.1016/j.gaceta.2016.03.007

Musitu-Ochoa, G., Suárez-Relinque, C., Del Moral-Arroyo, G., \& Villareal-González, M. E. (2015). El consumo de alcohol en adolescentes: el rol de la comunicación, el funcionamiento familiar, la autoestima y el consumo en la familia y amigos. Búsqueda, 2(14),45-61. Recuperado de https://rio.upo.es/xmlui/handle/10433/4378

Navarro-Oliva, E. I., Alonso-Castillo, M. M., Guzmán-Facundo, F. R., Álvarez-Bermúdes, J., \& Rodríguez-Puente, L. 
A. (2020). Uso de Facebook y persuasión interpersonal masiva: predictores del consumo de alcohol en jóvenes. SMAD Revista Electrónica Salud Mental, Alcohol y Drogas, 16(1), 1-8. Recuperado de https://dx.doi.org/10.11606/ issn.1806-6976.smad.2020.152351

Oliva-Delgado, A., Antolín, L. S., Estévez, R. M. C., \& Pascual, D. G. (2012). Activos del Barrio y Ajuste Adolescente. Psychosocial Intervention, 21(1), 17-27. Recuperado de http://dx.doi.org/10.5093/in2012v21n1a1

Organización de los Estados Americanos (OEA), Comisión Interamericana para el Control del Abuso de Drogas [ClCAD], Secretaria de Seguridad Multidimensional [SSM] (2019). Informe sobre uso de drogas en las Américas 2019. Recuperado de http://www.cicad.oas.org/main/pubs/Informe\%20sobre\%20el\%20consumo\%20de\%20drogas\%20 en\%20las\%20Am\%C3\%A9ricas\%202019.pdf

Organización Mundial de la Salud [OMS]. (2014). Global status report on alcohol and Health Alcohol 2014. Recuperado de https://apps.who.int/iris/bitstream/handle/10665/112736/9789240692763_eng.pdf

Organización Mundial de la Salud [OMS]. (2018). Global status report on alcohol and Health Alcohol 2018. Recuperado de https://apps.who.int/iris/bitstream/handle/10665/274603/9789241565639-eng.pdf?ua=1.2

Organización Panamericana de la Salud [OPS]. (2015). Informe de situación regional sobre el alcohol y la salud en las Américas. Recuperado de https://www.paho.org/hq/dmdocuments/2015/alcohol-Informe-salud-americas-2015. pdf

Ortiz-Peña, M. C, Navarro-Oliva, P. I., E., Echevarria-Cerda, F. J, Guzmán-Facundo, F. R., Pacheco-Pérez, L. A., \& Rodríguez-Puente, L. A. (2019). Apoyo social percibido a través de Facebook, familia y amigos y el consumo de alcohol en adolescentes. Health \& Addictions/Salud y Drogas, 19(2), 103-110. Recuperado de https://ojs.haaj.org/index.php/ haaj/article/view/448/pdf

Ovejero, B. M. M., \& Cardenal, H. V. (2015). Fortalezas humanas desde la perspectiva de género: un estudio exploratorio en población española. Revista Mexicana de Investigación Psicológica, 7(2), 72-92. Recuperado de https:// www.revistamexicanadeinvestigacionenpsicologia.com/index.php/RMIP/article/view/203

Pastor, A., Espelt, A., Villalbí, J. R., Moure, L., Fuentes, S., Shortt, N. K., ... \& Sureda, X. (2020). Disponibilidad y promoción de alcohol según la tipología de los locales y las condiciones socioeconómicas del área. Adicciones, 20(10). Recuperado de: http://ww.adicciones.es/index.php/adicciones/article/view/1367

Peltzer, K., \& Pengpid, S. (2016). Heavy drinking and social and health factors in university students from 24 low, middle income and emerging economy countries. Community Mental Health Journal, 52(2), 239-244. DOI 10.1007/ s10597-015-9925-x

Pérez, L. D. C., Miguelena, R., \& Diallo, A. F. (2016). La efectividad de la formación en ambientes virtuales de aprendizaje en la educación superior. Campus virtuales, 5(2), 10-17. Recuperado de http://www.uajournals.com/ojs/index. php/campusvirtuales/article/view/159/127

Pons, J., \& Buelga, S. (2011). Factores Asociados al consumo juvenil de alcohol: una revisión desde una perspectiva psicosocial y ecológica. Psychosocial Intervention, 20(1), 75-94. Recuperado de https://doi.org/10.5093/in2011v20n1a7

Reckdenwald, A., Ford, J. A., \& Murray B. N. (2016). Alcohol use in Emerging Adulthood: Can Moffitt's developmental theory helps us understand binge drinking among college students?. Journal of child \& adolescent substance abuse, 12(18). DOI:10.1080/1067828X.2015.1103347 
Reyes, A. R., Rodríguez Aguilar, L., Selene López, K., Guzmán, F. R., \& Magdalena Alonso, M. (2018). Estrés Laboral, Resiliencia y Consumo de Alcohol en Trabajadores de la Industria del Acero de Nuevo León, México. Health \& Addictions/Salud y Drogas, 18(1), 29-37. Recuperado de https://ojs.haaj.org/index.php/haaj/article/view/330

Reynaga-Ornelas, M. G., Fernández-Carrasco, M. P., Muñoz-Canul, I. C., \& Vera-Becerra, L. E. (2015). Percepción de comportamientos de riesgo en estudiantes universitarios del área de la salud. Acta Universitaria, 25(NE-1), 44-51. DOI: 10.15174/au.2015.763

Saiz, M. S., Chacón, R. F., Abejar, M. G., Parra, M. S., Valentín, M. D., \& Yubero, S. (2020). Perfil de consumo de drogas en adolescentes. Factores protectores. Medicina de Familia. SEMERGEN, 46(1), 33-40. Recuperado de https://doi. org/10.1016/j.semerg.2019.06.001

Sánchez, C. N. L. (2018). Conducta resiliente y abuso de alcohol en estudiantes de Psicología de la UMSA. Revista de Investigación Psicológica, 20, 11-27. Recuperado de: http://www.scielo.org.bo/pdf/rip/n20/n20_a03.pdf

Sánchez-Sosa, J.C., Villarreal-González, M.E., Ávila, M.E., Vera, A., \& Musito, G. (2014). Contextos de socialización y consumo de drogas ilegales en adolescentes escolarizados. Psychosocial Intervention, 23, 69-78. Recuperado de http://dx.doi.org/10.5093/in2014a7

Scoppetta, O., Martínez Ortiz, E., \& Martín, A. (2019). Percepción de riesgo e influencia normativa: asociación con el consumo de sustancias en estudiantes. Health \& Addictions/Salud y Drogas, 19(2), 8-18. Recuperado de https:// doi.org/10.21134/haaj.v19i2.427

Sharma, M., Anyimukwu, C., Kim, R. W., Nahar, V. K., \& Ford, A. (2018). Predictors of responsible Drinking or Abstinence among college students who Bing Drinking: A Multitheory Model Approach. The Journal of the American Osteopathic Association, 118(8), 519-530. DOI: 10.7556/jaoa.2018.120

Simons-Morton, B., Haynie, D., Liu, D., Chaurasia, A., Li, K., \& Hingson, R. (2016). The effect of residence, school status, work status, and social influence on the prevalence of alcohol use among emerging adults. Journal of studies on alcohol and drugs, 77(1), 121-132. Recuperado de https://doi.org/10.15288/jsad.2016.77.121

Simons-Morton, B., Haynie, D., Liu, D., Chaurasia, A., Li, K., \& Hingson, R. (2016). The effect of residence, school status, work status, and social influence on the prevalence of alcohol use among emerging adults. Journal of studies on alcohol and drugs, 77(1), 121-132. Recuperado de https://doi.org/10.15288/jsad.2016.77.121

Smilkstein, G. (1978). The family APGAR: A proposal for a family function test and its uses by physicians. The Journal of Family Practice, 6, 1231-1239. Recuperado de https://mdedge-files-live.s3.us-east-2.amazonaws.com/files/ s3fs-public/jfp-archived-issues/1978-volume_6-7/JFP_1978-06_v6_i6_the-family-apgar-a-proposal-for-a-family.pdf

Suárez-Zozaya, M. H. (2018). Génesis de la juventud de los estudiantes universitarios. Perfiles educativos, 40(159), 177-191. Recuperado de http://www.scielo.org.mx/pdf/peredu/v40n159/0185-2698-peredu-40-159-177.pdf

Sudhinaraset, M., Wigglesworth, C., \& Takeuchi, D. T. (2016). Social and cultural contexts of alcohol use: Influences in a social-ecological framework. Alcohol Research: Current Reviews, 38(1), 35-45. Recuperado de https://pdfs.semanticscholar.org/2ea6/b2d315fb76dbca947f276d12060575eb0a7f.pdf?_ga=2.129304388.930366041.1573758010385897246.1565281660

Tovolacci, M. P., Boerg, E., Richard, L., Meyrignac, G., Dechelotte, P., \& Ladner, J. (2016). Prevalence of binge drinking and associated behaviors among 3286 college students in France. Public Health, 16(178), 1-9. DOI: 10.1186/s12889016-2863-x 
Trigueros, R., Álvarez, J. F., Aguilar-Parra, J. M., Alcaraz-Ibáñez, M., y Rosado, A. (2017). Validación y adaptación española de la escala de resiliencia en el contexto deportivo (ERCD). Psychology, Society, \& Education, 9(2), 311-324. DOI: $10.25115 /$ psye.v9i2.864

Uroz-Olivares, J., Charro-Baena, B., Prieto-Úrsua, M., \& Meneses-Falcón, C. (2018). Estructura familiar y consumo de alcohol en adolescentes. Health \& Addictions/Salud y Drogas, 18(1), 107-118. Recuperado de https://doi. org/10.21134/haaj.v18i1.364

Valdez, P. E., Pech, P. D., \& Lira, M.J. (2018). Expectativas como predictores del consumo de alcohol en universitarios. Rev. Latinoamericana de Medicina Conductual, 8(2), 43-49. Recuperado de http://www.revistas.unam.mx/index. php/rlmc/article/view/67351/60047

Villacé, M. B., Fernández, A. R., \& Da Costa Junior, M. L. (2013). Consumo de alcohol según características sociodemográficas en jóvenes de 18 a 24 años. Revista Latino-Americana de Enfermagem, 21(5), 1144-1150. Recuperado de https://www.scielo.br/pdf/rlae/v21n5/es_0104-1169-rlae-21-05-1144.pdf

Walker, L. O., \& Avant, K. C. (2019). Strategies for theory construction in nursing (6a. ed). Boston, USA: Pearson/ Prentice Hall.

Yi, S., Ngin, C., Peltzer, K., \& Pengpid, S. (2017). Health and behavioral factors associated with binge drinking among university students in nine ASEAN countries. Substance abuse treatment, prevention, and policy, 12(1), 32. DOI $10.1186 / \mathrm{s} 13011-017-0117-2$

Yuksek, D. A., \& Solakoglu, O. (2016). The relative influence of parental attachment, peer attachment, school attachment, and school alienation on delinquency among high school students in Turkey. Deviant Behavior, 37(7), 723-747. DOI: 10.1080/01639625.2015.1062683

Yurasek, A. M., Brick, L., Nestor, B., Hernandez, L., Graves, H., \& Spirito, A. (2019). The Effects of Parent, Sibling and Peer Substance Use on Adolescent Drinking Behaviors. Journal of Child and Family Studies, 28(1), 73-83. Recuperado de https://doi.org/10.1007/s10826-018-1251-9

Zhang, X., Hatcher, B., Clarkson, L., Holt, J., Bagchi, S., Kanny, D., \& Brewer, R. D. (2015). Peer Reviewed: Changes in Density of On-Premises Alcohol Outlets and Impact on Violent Crime, Atlanta, Georgia, 1997-2007. Preventing chronic disease, 12. Recuperado de https://www.ncbi.nlm.nih.gov/pmc/articles/PMC4454408/pdf/PCD-12-E84.pdf 